\title{
Effect of Exhausting Exercise on Oxidative Stress in Health, Hemodialysis and Professional Sport
}

\author{
Knap Bojan"1,2, Prezelj Marija3 ${ }^{3}$ Večerić-Haler Željka',2 \\ ${ }^{1}$ Medical Faculty University of Ljubljana, Ljubljana, Slovenia; ${ }^{2}$ Department of Nephrology, University Medical Centre \\ Ljubljana, Ljubljana, Slovenia; ${ }^{3}$ Institute of Clinical Chemistry and Biochemistry, University Medical Centre \\ Ljubljana, Ljubljana, Slovenia
}

Correspondence to: Knap Bojan, bojan.knap@kclj.si

Keywords: Hemodialysis, Oxidative Stress, Strenuous Exercise, Rowers, Oxidative Stress Athletes, Oxidative Stress Dialysis

Received: August 28, $2019 \quad$ Accepted: November 11, $2019 \quad$ Published: November 14, 2019

Copyright $\odot 2019$ by author(s) and Scientific Research Publishing Inc.

This work is licensed under the Creative Commons Attribution International License (CC BY 4.0).

http://creativecommons.org/licenses/by/4.0/

\section{(c) () Open Access}

\section{ABSTRACT}

The aim of presented study is to compare parameters of oxidative stress in untrained volunteers, patients with chronic, non-motor related disease (dialysis patients) and professional athletes before and after exhaustive exercise. 40 subjects participated in the study: 14 healthy, untrained subjects, 12 hemodialysis patients and 14 professional rowers. Superoxide dismutase (SOD), catalase (CAT), glutathione peroxidase (GPx) and non-transferrin bound iron (NTBI) were determined before and after exercise. Dialysis patients have increased oxidative stress at rest with highest NTBI, and show adaptation with increased values of GPx and decreased SOD. Professional athletes have low level of oxidative stress at basic circumstances with lowest NTBI, SOD, CAT and GPx as compared to untrained volunteers and dialysis patients. After strenuous exercise elevation of antioxidative enzymes is observed only in athletes, but not in untrained and dialysis patients. Due to limited antioxidative capacity, extreme physical effort is probably not recommended to dialysis patients and untrained people.

\section{INTRODUCTION}

There is a delicate equilibrium between the antioxidant defense system and free radicals in all living organisms, including humans. The breaking of this balance, frequently named oxidative stress, may induce cellular damage. Reactive oxygen species (ROS) is an abbreviation for a group of reactive molecules and free radicals extracted from molecular oxygen, such as superoxide anion $\left(\mathrm{O}_{2} \cdot^{-}\right)$and hydrogen peroxide $\left(\mathrm{H}_{2} \mathrm{O}_{2}\right)$. Normally, there are endogenous antioxidants present in organism. These are the enzymes catalase (CAT), superoxide dismutase (SOD), and glutathione peroxidase (GPx), which neutralize ROS [1, 2]. On the other hand, non-transferrin bound iron (NTBI) serves as indicator of oxidative stress. It is a powerful 
promotor of free radical damage and highly toxic to biological systems, resulting in oxidative damage to proteins, lipids and DNA [3].

The role of oxidative stress has been postulated in many diseases and pathologic conditions, including atherosclerosis, inflammatory conditions, certain cancers, the process of aging, as well as end stage kidney disease $[1,4]$. There is good evidence indicating that uremia in general is associated with enhanced oxidative stress, and the treatment of uremic patients with hemodialysis has been suggested as particularly contributing to oxidative stress and reduced antioxidant levels in these patients $[4,5]$. On the other "extreme" end, elite sports engagement is also a potentially strong stimulus of oxidative stress that leads to the large recruitment of antioxidative defense [6].

Average physical capabilities of hemodialysis patients worldwide, as well as in our dialysis units, are low $[7,8]$. Although exercise was shown to have pleiotropic beneficial therapeutic potential in patients with chronic kidney diseases [8-11], it has been postulated that intensive physical training generates free radicals resulting in possible additional cell damage [12-15]. The oxidative stress in hemodialysis patients has been extensively studied [4], however, there is still no consensus about the proper level of physical activity regarding additional expenditure of antioxidative "machinery" to be beneficial and therefore safely recommended to this specific population.

The aim of our study was to compare the antioxidant status of healthy individuals, patients with end-stage kidney failure, and professional athletes (rowers) before and after exercise, in this way confronting the averagely trained population with people supposed to have very decreased (hemodialysis patients) and increased (rowers) antioxidant capacity.

\section{MATERIALS AND METHODS}

\subsection{Subjects}

40 subjects participated in the study, including 12 patients with end-stage kidney disease (treated with hemodialysis), 14 healthy subjects, and 14 elite rowers. The study protocol was approved by the National Ethics Board. The experiments in this study were explained to all participants and, before obtaining human materials, informed consent was obtained from the subjects.

There were ten males and two females in the group of dialysis patients. The age of patients ranged 20 - 50 years, average 35 years. Exclusion criteria in hemodialysis patients were the next: concomitant comorbidities, including ischemic cardiac disease, acute illness (febrile state, infection), high blood pressure, hypertrophy of left ventricle, malignant disease, severe anemia, autoimmune disease, immunosuppressive treatment. The patients had regularly been on renal replacement therapy with hemodialysis, three times per week, for on average nine years dialysis vintage. A blood analysis was taken in the morning, in hemodialysis patients on the day without dialysis procedure.

The group of volunteers was comprised of 14 healthy subjects, seven women and seven male, average 25 years old. Excluding criteria for control group were the following: chronic illness, medicine therapy, professional sport activity, pregnancy, anemia and higher body mass index than thirty.

Slovenia's national rowing team is comprised of 14 elite rowers (all males) who regularly compete in world championships and the Olympic Games. The age of rowers was between 19 and 35 years, average 22 years.

\subsection{Methods}

Blood samples were taken in the morning for the rest measurements and five minutes after each exercise procedure. Blood data analyses were made in Institute of Clinical Chemistry and Biochemistry, University Medical Centre Ljubljana. Venous blood samples were obtained from participants of the study into vacutainer tubes two days before exercise, when no exercise had been performed in the previous twenty-four hours and right after intensive exercise. GPx, CAT and SOD in erythrocytes were measured using the Randox reagent kit (Randox Laboratories, Crumlin, UK) by a Hitachi Modular biochemical analyzer (Roche Diagnostics, Mannheim, Germany). A Carry 4E atomic absorption spectrometer (Varian, Inc.) was used for plasma selenium (Se) determination [16]. Non transferrin bound iron (NTBI) was measured co- 
lorimetrically in the ultrafiltrate using Hitachi Modular Analyzer (Roche Diagnostics) according to the FerroZine assay method [17].

\subsection{Exercise Protocol}

Exercise stress test was made in Diagnostic Exercise Laboratory, Faculty of Sport, University of Ljubljana in 2018. Classical progressive cycle ergometer protocol for detection of ischemic cardiac disease was employed. Healthy volunteers were loaded with starting exercise intensity of fifty Watts for three minutes, which was progressively increased for fifty Watts at every further step with three minutes duration until exhaustion. Exercise procedure for hemodialysis patients was adapted to patient physical capability. They started on intensity of thirty Watts and progress intensity every three minutes for thirty Watts until exhaustion.

Rowers exercised for nine minutes using a rowing cycle ergometer. The first three minutes the rowers exercised at anaerobic threshold intensity, in the next three minutes intensity was increased and during the last three minutes maximum intensity was reached. At the end rowers should generally achieve maximum heart rates.

The data were analyzed using standard statistical methods and the Mann-Whitney test was used for comparison between the groups; $\mathrm{p}<0.05$ was considered significant. Statistical analyses were performed using a statistical software package Statgraphics centurion XVII.

\section{RESULTS}

The results of basic characteristics of study groups and observed biochemistry parameters (glucose, phosphate, urate) are presented in Figure 1 and Table 1, respectively.

Significant decrease of glucose concentration after strenuous exercise was observed in healthy volunteers, and the opposite in rowers $(\mathrm{p}<0.01)$. There were no significant changes in urate concentrations before and after exercise in all groups. The concentration of phosphate increased significantly after exercise in the groups of healthy and rowers $(\mathrm{p}<0.01)$, showing no significant changes in the group of dialysis patients.

In healthy volunteers the activities of GPx $(0.86 \pm 0.15$ vs $0.89 \pm 0.18 ; \mathrm{p}=0.52)$, concentrations of Se $(65.07 \pm 10.16$ vs $72.14 \pm 11.13 ; \mathrm{p}=0.09)$ and NTBI $(1.33 \pm 0.93$ vs $1.71 \pm 1.16 ; \mathrm{p}=0.34)$ increased, while the activity of SOD decreased $(19.38 \pm 3.91$ vs $18.67 \pm 4.23$; $\mathrm{p}=0.64)$ after strenuous exercise, although statistical significance was not reached in either case.

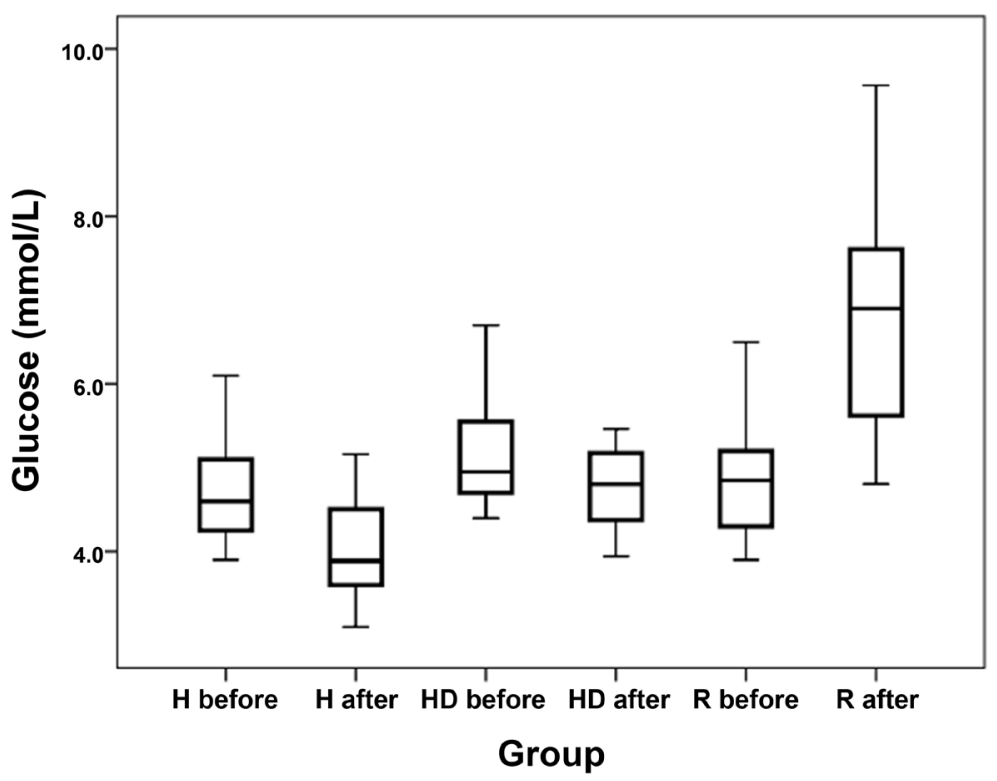



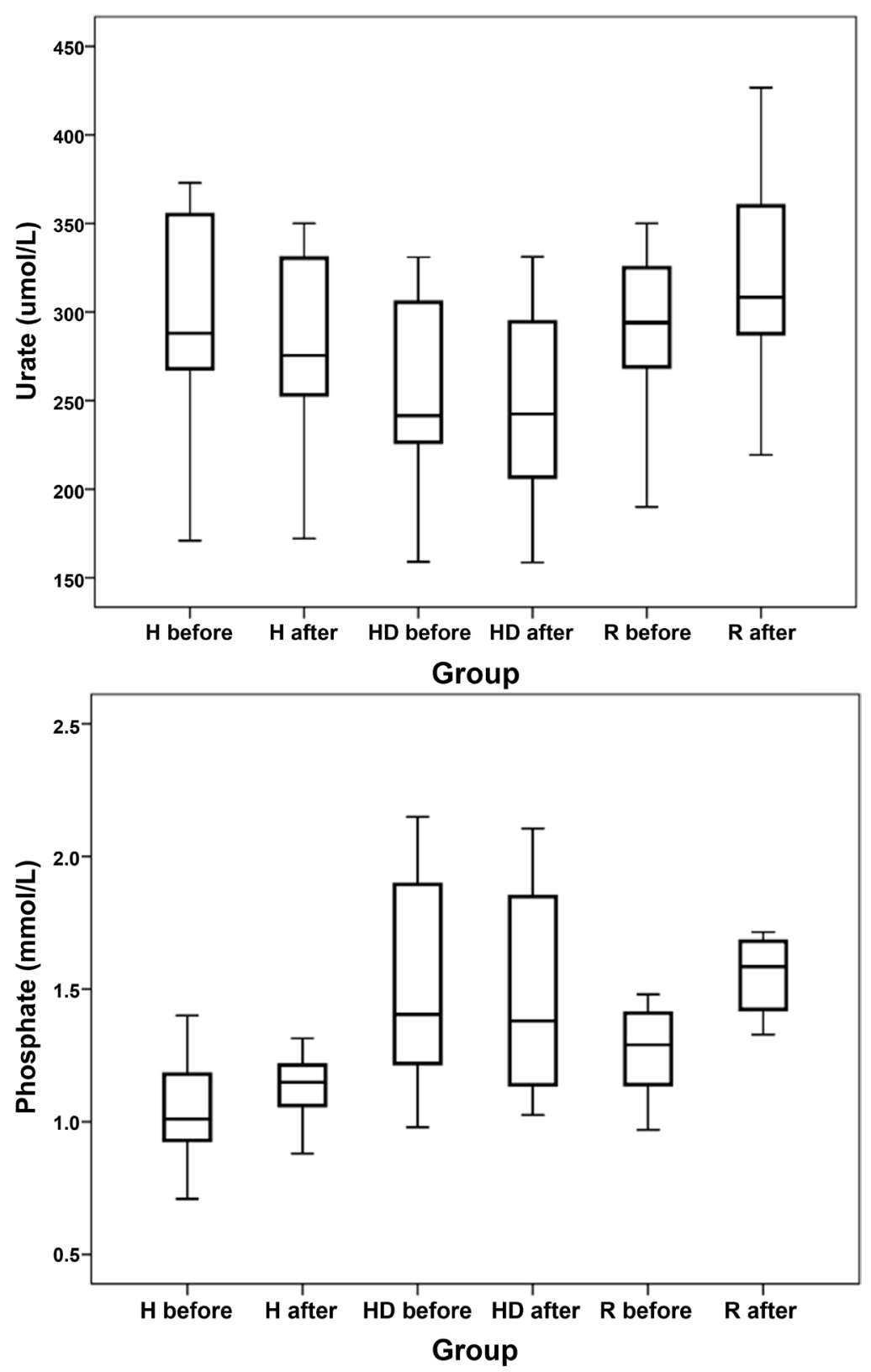

Figure 1. Observed laboratory parameters (glucose, urate and phosphate) before and after exercise in healthy volunteers $(\mathrm{H})$, dialysis patients (HD) and rowers (R). The boxes represent the $25^{\text {th }}-75^{\text {th }}$ percentile limits, with the median value represented by a line in the box.

Table 1. Basic characteristics in healthy volunteers (healthy), dialysis patients (HD patients) and rowers.

\begin{tabular}{cccc}
\hline & HEALTHY & HD PATIENTS & ROWERS \\
\hline AGE & $25.6 \pm 3.9$ & $35.6 \pm 10.5$ & $22.14 \pm 4.95$ \\
GENDER & $7 \mathrm{~W} .7 \mathrm{M}$ & $2 \mathrm{~W} .10 \mathrm{M}$ & $14 \mathrm{M}$ \\
\hline
\end{tabular}


In hemodialysis patients the concentration of Se $(56.0 \pm 10.63$ vs $61.08 \pm 11.38 ; \mathrm{p}=0.27)$ and NTBI $(1.5 \pm 0.67$ vs $1.82 \pm 0.91 ; \mathrm{p}=0.33)$ increased, while the activities of GPx $(1.05 \pm 0.23$ vs $1.03 \pm 0.23 ; \mathrm{p}=$ $0.83)$ and SOD $(14.63 \pm 2.66$ vs $14.28 \pm 2.36 ; \mathrm{p}=0.73)$ decreased after strenuous exercise, the differences were not statistically significant.

Except from higher level of basic GPx activity $(0.86 \pm 0.15$ vs $1.05 \pm 0.23 ; p=0.01)$ there were no marked differences in activities of other observed pertinent of oxidative stress in healthy volunteers' vs hemodialysis patients before or after exercise. Values of CAT were the highest in dialysis patients at rest and the lowest at rowers (Table 2). CAT values increase significantly at rowers after exercise in comparison to healthy and patients.

In basic circumstances rowers had much higher concentrations of Se $(106.1 \pm 17.8 \mu \mathrm{g} / \mathrm{l} \mathrm{vs} 65.07 \pm$ $10.16 \mu \mathrm{g} / \mathrm{l} ; \mathrm{p}<0,001)$ and lower levels of both NTBI $(0.56 \pm 0.38$ vs $1.33 \pm 0.93 ; \mathrm{p}<0.009)$ and SOD activity $(19.38 \pm 3.91$ vs $14.09 \pm 0.92 ; \mathrm{p}<0.001)$ as compared to healthy volunteers, with no significant difference in activity of GPx $(0.86 \pm 0.15$ vs $0.76 \pm 0.16 ; \mathrm{p}=0.09)$.

After the exercise protocol drop of SOD activity (14.09 \pm 0.92 vs $13.55 \pm 0.66 ; \mathrm{p}=0.08)$, significant increase in NTBI $(0.57 \pm 0.35$ vs $3.58 \pm 2.52 ; \mathrm{p}<0.001)$ and increase in Se $(106.1 \pm 17.8$ vs $122.36 \pm 18.1 ; \mathrm{p}$ $=0.02$ ) were detected in rowers. There was no difference in the activity of GPx before vs after exercise in rowers $(0.76 \pm 0.16$ vs $0.74 \pm 0.17 ; \mathrm{p}=0.75)$. Values of Se after exercise were significantly higher in rowers $(122.36 \pm 18 \mu \mathrm{g} / \mathrm{l}$ and $72.14 \pm 11.1 \mu \mathrm{g} / \mathrm{l} ; \mathrm{p}<0.001)$ compared to healthy volunteers.

\section{DISCUSSION}

Our study investigated and compared the response of acute exhaustive, aerobic physical exercise on oxidative stress damage in hemodialysis patients in comparison to the response in healthy, untrained volunteers and professional athletes (rowers).

The results of this study show that dialysis patients when compared to healthy, albeit untrained people,

Table 2. Important values of study oxidative stress parameters before and after exercise in healthy volunteers (healthy), dialysis patients (HD patients) and rowers. Legend: GPx-glutathion peroxidase, SOD-superoxide dismutase, Se-selenium, NTBI-non-transferrin binding iron, CAT-catalase, ns-non significant $\mathrm{p}>0.05$.

\begin{tabular}{cccccc}
\hline BEFORE EXERCISE & $\begin{array}{c}\text { HEALTHY } \\
(\mathbf{n}=14)\end{array}$ & $\begin{array}{c}\text { HD PATIENTS } \\
(\mathbf{n}=12)\end{array}$ & $\mathbf{p}$ & $\begin{array}{c}\text { ROWERS } \\
(\mathbf{n}=14)\end{array}$ & $\mathbf{p}$ \\
\hline GPx (ukat/gHb) & $0.86 \pm 0.15$ & $1.05 \pm 0.23$ & 0.01 & $0.76 \pm 0.16$ & $\mathrm{~ns}$ \\
Se (ug/L) & $65.07 \pm 10.16$ & $56.00 \pm 10.63$ & $\mathrm{~ns}$ & $106.143 \pm 17.82$ & $<0.001$ \\
SOD (ukat/gHb) & $19.38 \pm 3.91$ & $14.63 \pm 2.66$ & $\mathrm{~ns}$ & $14.09 \pm 0.92$ & $<0.001$ \\
NTBI (umol/L) & $1.33 \pm 0.93$ & $1.50 \pm 0.67$ & $\mathrm{~ns}$ & $0.568 \pm 0.38$ & 0.009 \\
CAT (mkat/gHb)) & $2.64 \pm 0.39$ & $2.78 \pm 0.38$ & $\mathrm{~ns}$ & $1.72 \pm 0.31$ & $<0.001$ \\
\hline AFTER EXERCISE & HEALTHY & HD PATIENTS & $\mathbf{p}$ & ROWERS & $\mathrm{p}$ \\
& $(\mathbf{n}=14)$ & $(\mathbf{n}=12)$ & & $(\mathbf{n}=14)$ & $\mathrm{ns}$ \\
GPx (ukat/gHb) & $0.89 \pm 0.18$ & $1.03 \pm 0.23$ & $\mathrm{~ns}$ & $0.74 \pm 0.17$ & $<0.001$ \\
Se (ug/L) & $72.14 \pm 11.13$ & $61.08 \pm 11.38$ & $\mathrm{~ns}$ & $122.357 \pm 18.12$ & $<0.001$ \\
SOD (ukat/gHb) & $18.67 \pm 4.23$ & $14.28 \pm 2.36$ & $\mathrm{~ns}$ & $13.55 \pm 0.66$ & 0.02 \\
NTBI (umol/L) & $1.71 \pm 1.16$ & $1.82 \pm 0.91$ & $\mathrm{~ns}$ & $3.58 \pm 2.52$ & $<0.001$ \\
\hline CAT (mkat/gHb) & $2.71 \pm 0.45$ & $2.81 \pm 0.567$ & $\mathrm{~ns}$ & $2.14 \pm 0.41$ &
\end{tabular}


have increased oxidative stress at rest with highest NTBI, and show adaptation with increased basic values of GPx and moderately decreased SOD. Contrary, professional athletes have low level of oxidative stress at basic circumstances with lowest concentrations of NTBI, SOD, CAT and GPx as compared to untrained volunteers and dialysis patients. According to highest NTBI after exercise (and highest level of exercise intensity), athletes reach highest level of oxidative stress after strenuous workout, which does not deplete their antioxidative machinery as observed with almost unremarkable changes in SOD and GPX and significant elevation of CAT. Elevation of antioxidative enzymes after strenuous exercise was not observed in untrained and dialysis patients.

There is much evidence to show that low intensity exercise training enables improved physical performance and functioning among patients with end stage kidney disease on dialysis treatment [12]. The majority of physical exercise trials performed so far tested low to moderate intensity interventions performed during the hemodialysis session and consisted either of aerobic exercise (cycling) or resistance training of some muscular groups against elastic bands, or a combination thereof [8-10, 17-19]. On the other hand, there are no data on effects of strenuous exercise in dialysis patients, especially in the context of known contribution of hemodialysis on increased oxidative stress $[4,17,18]$. Dialysis is associated with a deterioration of physical function and mental status $(6,7,16$ - 19). Muscle function [19] and exercise capacity [20] are significantly lower in hemodialysis patients, presenting a peak oxygen consumption that is considerably lower (less than 50\%) compared to their healthy sedentary peers.

Antioxidant enzymes like SOD are present in the erythrocytes of all mammals and represent the first defensive role against ROS [1,2]. SOD catalyzes dismutation of the superoxide radicals with hydrogen peroxide $\left(\mathrm{H}_{2} \mathrm{O}_{2}\right)$ formation, which is severely cytotoxic, and is further neutralized by CAT or GPx. With its high affinity and low capacity, GPx acts especially important part at low physiological fluxes of $\mathrm{H}_{2} \mathrm{O}_{2}$. The significantly higher activity of GPx in basic circumstances with low SOD activity is most probably the physiologic answer to high $\mathrm{H}_{2} \mathrm{O}_{2}$ in presence of constantly high NTBI in dialysis patients. The highest NTBI concentration in dialysis patient group is the consequence of the extremely high ferritin concentration levels (not shown) in this group and is at least partly the effect of iron supplementation provided to the patients routinely receiving ferric saccharate. Similar constellation of oxidative enzymes and NTBI were already described by us and others and indicate adaptation to chronic oxidative stress in end-stage renal diseases $[6,13,17,18]$. The values of Se, a constitutional part of GPx, are significantly lower in patients as compared to untrained individuals and rowers and are in accordance with so far published results in patients with end stage kidney disease [21]. Higher values in rowers could be associated with extensive (or even excessive) Se and vitamin supplementation that is often provided to professional sportsmen. The observation of significant increase in the blood phosphate concentration in rowers after strenuous exercise is most probably the reflection of maximal intensity level of workout reached in this group associated with extensive consumption of ATP. When one molecule of ATP is used in the muscle contraction process, it is hydrolyzed to ADP, adenosine diphosphate, and an inorganic phosphate. However, higher glucose concentrations in rowers after strenuous exercise remain unexplained.

In our study the exhaustive physical exercise was unable to increase plasma antioxidant enzymes levels in healthy volunteers and hemodialysis patients. In the same time plasma NTBI and CAT concentration were significantly increased after acute physical exercise in professional athletes. These data suggest that intense interdialytic aerobic exercise was unable to increase the circulating concentration of antioxidative enzymes in hemodialysis, as well as in healthy, untrained volunteers. This observation likely indicates absence of adaptation to oxidative stress provoked by acute, strenuous aerobic exercise. Contrary, regular physical training seems to reduce the oxidative stress associated with exercise, such that trained athletes show less evidence of oxidative stress for given bout of exercise and an enhanced defense system in relation to untrained subjects, which was already observed by other authors as well $[13,17,18]$.

At present, mild to moderate intensity physical exercise is recommended for healthy untrained people and hemodialysis patients due to their numerous chronic beneficial effects, which include increased aerobic capacity, muscle strength, production of antioxidants, control of blood pressure and decreased fatigue [8-10]. The eventual benefits of exhaustive trainings in dialysis population are for now, unknown. 
In accordance with our results, it is possible that acute exhaustive exercise could have detrimental effects in the short term, such as increased oxidative stress and decreased production of antioxidant enzymes. The question whether this could lead to aggravation of clinical condition of dialysis patients or have noxious effects on healthy, untrained persons remains to be answered.

\section{CONCLUSIONS}

According to this study analysis, patients show comparable oxidative stress response to acute, strenuous exercise as healthy, otherwise untrained persons. In comparison with patients and untrained individuals, professional athletes show changes probably associated with adaptation to strenuous exercise.

Acute strenuous exercise might be related to increased oxidative stress consequences in diseased or untrained people due to lack of adaptive mechanisms and should not be recommended until results of further studies are obtained.

\section{CONFLICTS OF INTEREST}

Authors declare no conflicts of interest.

\section{REFERENCES}

1. Liu, Z., Ren, Z., Zhang, J., Chuang, C.C., Kandaswamy, E., et al. (2018) Role of ROS and Nutritional Antioxidants in Human Diseases. Frontiers in Physiology, 9, 477. https://doi.org/10.3389/fphys.2018.00477

2. Lemire, J., Alhasawi, A., Appanna, V.P., Tharmalingam, S. and Appanna, V.D. (2017) Metabolic Defence against Oxidative Stress: The Road Less Travelled So Far. Journal of Applied Microbiology, 123, 798-809. https://doi.org/10.1111/jam.13509

3. Patel, M. and Ramavataram, D.V. (2012) Non Transferrin Bound Iron: Nature, Manifestations and Analytical Approaches for Estimation. Indian Journal of Clinical Biochemistry, 27, 322-332. https://doi.org/10.1007/s12291-012-0250-7

4. Modaresi, A., Nafar, M. and Sahraei, Z. (2015) Oxidative Stress in Chronic Kidney Disease. Iranian Journal of Kidney Diseases, 9, 165-79.

5. Liakopoulos, V., Roumeliotis, S., Gorny, X., Dounousi, E. and Mertens, P.R. (2017) Oxidative Stress in Hemodialysis Patients: A Review of the Literature. Oxidative Medicine and Cellular Longevity, 2017, Article ID: 3081856. https://doi.org/10.1155/2017/3081856

6. Hadžović-Džuvo, A., Valjevac, A., Lepara, O., Pjanić, S., Hadžimuratović, A. and Mekić, A. (2014) Oxidative Stress Status in Elite Athletes Engaged in Different Sport Disciplines. Bosnian Journal of Basic Medical Sciences, 14, 56-62. https://doi.org/10.17305/bjbms.2014.2262

7. Persic, V., Slonjšak, B., Pajek, J. and Buturović Ponikvar, J. (2018) Prevalence of Impaired Physical Mobility in Dialysis Patients: A Single Centre Cross-Sectional Study. 12 th International Congress of ISHD, Cartagena, 18-21 April 2018.

8. Bučar Pajek, M., et al. (2016) Six-Minute Walk Test in Renal Failure Patients: Representative Results, Performance Analysis and Perceived Dyspnea Predictors. PLoS ONE, 11, e0150414. https://doi.org/10.1371/journal.pone.0150414

9. Storer, T.W., Casaburi, R., Sawelson, S. and Kopple, J.D. (2005) Endurance Exercise Training during Haemodialysis Improves Strength, Power, Fatigability and Physical Performance in Maintenance Haemodialysis Patients. Nephrology Dialysis Transplantation, 20, 1429-1437. https://doi.org/10.1093/ndt/gfh784

10. Ouzouni, S., Kouidi, E., Sioulis, A., Grekas, D. and Deligiannis, A. (2009) Effects of Intradialytic Exercise Training on Health-Related Quality of Life Indices in Hemodialysis Patients. Clinical Rehabilitation, 23, 53-63. 
https://doi.org/10.1177/0269215508096760

11. Smart, N., McFarlane, J. and Cornelissen, V. (2013) The Effect of Exercise Therapy on Physical Function, Biochemistry and Dialysis Adequacy in Haemodialysis Patients: A Systematic Review and Meta-Analysis. Open Journal of Nephrology, 3, 25-36. https://doi.org/10.4236/ojneph.2013.31005

12. Manfredini, F., Mallamaci, F., D’Arrigo, G., Baggetta, R., Bolignano, D., et al. (2017) Exercise in Patients on Dialysis: A Multicenter, Randomized Clinical Trial. Journal of the American Society of Nephrology, 28, 1259-1268. https://doi.org/10.1681/ASN.2016030378

13. de Sousa, C.V., Sales, M.M., Rosa, T.S., Lewis, J.E., de Andrade, R.V., et al. (2017) The Antioxidant Effect of Exercise: A Systematic Review and Meta-Analysis. Sports Medicine, 47, 277-293.

https://doi.org/10.1007/s40279-016-0566-1

14. Barcellos, F.C., Santos, I.S., Umpierre, D., Bohlke, M. and Hallal, P.C. (2015) Effects of Exercise in the Whole Spectrum of Chronic Kidney Disease: A Systematic Review. Clinical Kidney Journal, 8, 753-765.

https://doi.org/10.1093/ckj/sfv099

15. Heiwe, S. and Jacobson, S.H. (2014) Exercise Training in Adults with CKD: A Systematic Review and Meta-Analysis. American Journal of Kidney Diseases, 64, 383-393. https://doi.org/10.1053/j.ajkd.2014.03.020

16. https://www.randox.com/diagnostic-reagents

17. Prezelj, M. and Knap, B. (2010) Automated Assay for Non-Transferrin-Bound Iron in Serum Samples. Clinical Chemistry and Laboratory Medicine, 48, 1427-1432. https://doi.org/10.1515/CCLM.2010.287

18. Chakrabarti, C.L. (1968) The Atomic Absorption Spectroscopy of Selenium. Analytica Chimica Acta, 42, 379-387. https://doi.org/10.1016/S0003-2670(01)80329-9

19. Böhm, J., Monteiro, M.B., Andrade, F.P., Veronese, F.V. and Thomé, F.S. (2017) Acute Effects of Intradialytic Aerobic Exercise on Solute Removal, Blood Gases and Oxidative Stress in Patients with Chronic Kidney Disease. Brazilian Journal of Nephrology, 39, 172-180. https://doi.org/10.5935/0101-2800.20170022

20. Knap, B., Prezelj, M., Buturović-Ponikvar, J., Ponikvar, R. and Bren, A.F. (2009) Antioxidant Enzymes Show Adaptation to Oxidative Stress in Athletes and Increased Stress in Hemodialysis Patients. Therapeutic Apheresis and Dialysis, 13, 300-305. https://doi.org/10.1111/j.1744-9987.2009.00728.x

21. Leal, V.O., Stockler-Pinto, M.B., Farage, N.E., Aranha, L.N., Fouque, D., Anjos, L.A. and Mafra, D. (2011) Muscle Function, Handgrip Strength and Its Dialysis Determinants in Hemodialysis Patients. Nutrition, 27, 1125-1129. https://doi.org/10.1016/j.nut.2010.12.012 